\title{
GENERALIZED RAINBOW CONNECTION OF GRAPHS AND THEIR COMPLEMENTS
}

\author{
Xueliang Li $^{1}$, Colton Magnant ${ }^{2}$ \\ Meiqin Wei ${ }^{1}$ AND XiaOyu Zhu ${ }^{1}$ \\ ${ }^{1}$ Center for Combinatorics and LPMC \\ Nankai University, Tianjin 300071, China \\ ${ }^{2}$ Department of Mathematical Sciences \\ Georgia Southern University \\ Statesboro, GA 30460-8093, USA \\ e-mail: lxl@nankai.edu.cn \\ cmagnant@georgiasouthern.edu \\ weimeiqin8912@163.com \\ zhuxy@mail.nankai.edu.cn
}

\begin{abstract}
Let $G$ be an edge-colored connected graph. A path $P$ in $G$ is called $\ell$-rainbow if each subpath of length at most $\ell+1$ is rainbow. The graph $G$ is called $(k, \ell)$-rainbow connected if there is an edge-coloring such that every pair of distinct vertices of $G$ is connected by $k$ pairwise internally vertex-disjoint $\ell$-rainbow paths in $G$. The minimum number of colors needed to make $G(k, \ell)$-rainbow connected is called the $(k, \ell)$-rainbow connection number of $G$ and denoted by $r c_{k, \ell}(G)$. In this paper, we first focus on the $(1,2)$-rainbow connection number of $G$ depending on some constraints of $\bar{G}$. Then, we characterize the graphs of order $n$ with $(1,2)$-rainbow connection number $n-1$ or $n-2$. Using this result, we investigate the NordhausGaddum-Type problem of $(1,2)$-rainbow connection number and prove that $r c_{1,2}(G)+r c_{1,2}(\bar{G}) \leq n+2$ for connected graphs $G$ and $\bar{G}$. The equality holds if and only if $G$ or $\bar{G}$ is isomorphic to a double star.
\end{abstract}

Keywords: $\ell$-rainbow path, $(k, \ell)$-rainbow connected, $(k, \ell)$-rainbow connection number.

2010 Mathematics Subject Classification: 04C15, 05C40.

\section{REFERENCES}


[1] E. Andrews, E. Laforge, C. Lumduanhom and P. Zhang, On proper-path colorings in graphs, J. Combin. Math. Combin. Comput. 97 (2016) 189-207.

[2] V. Borozan, S. Fujita, A. Gerek, C. Magnant, Y. Manoussakis, L. Montero and Zs. Tuza, Proper connection of graphs, Discrete Math. 312 (2012) 2550-2560. doi:10.1016/j.disc.2011.09.003

[3] J.A. Bondy and U.S.R. Murty, Graph Therory (GTM 244, Springer-Verlag, New York, 2008).

[4] G. Chartrand, S. Devereaux and P. Zhang, Color-connected graphs and informationtransfer paths, Ars Combin., to appear.

[5] G. Chartrand, G.L. Johns, K.A. McKeon and P. Zhang, Rainbow connection in graphs, Math. Bohem. 133 (2008) 85-98.

[6] S. Devereaux, G.L. Johns and P. Zhang, Color connection in graphs intermediate to proper and rainbow connection, J. Combin. Math. Combin. Comput., to appear.

[7] S. Devereaux and P. Zhang, k-rainbow colorings in graphs, manuscript.

[8] J.L. Fouquet and J.L. Jolivet, Strong edge-coloring of graphs and applications to multi-k-gons, Ars Combin. 16A (1983) 141-150.

[9] M. Krivelevich and R. Yuster, The rainbow connection of a graph is (at most) reciprocal to its minimum degree, J. Graph Theory 63 (2010) 185-191. doi:10.1002/jgt.20418

[10] X. Li and C. Magnant, Properly colored notions of connectivity - a dynamic survey, Theory Appl. Graphs 0(1) (2015) Article 2. doi:10.20429/tag.2015.000102

[11] X. Li, C. Magnant, M. Wei and X. Zhu, Distance proper connection of graphs (2016) arXiv:1606.06547 [math.CO]

[12] X. Li and Y. Shi, Rainbow connection in 3-connected graphs, Graphs Combin. 29 (2013) 1471-1475. doi:10.1007/s00373-012-1204-9

[13] X. Li, Y. Shi and Y. Sun, Rainbow connections of graphs: A survey, Graphs Combin. 29 (2013) 1-38. doi:10.1007/s00373-012-1243-2

[14] X. Li and Y. Sun, Rainbow Connections of Graphs (Springer Briefs in Math., Springer, New York, 2012).

Received 26 July 2016

Revised 26 November 2016

Accepted 29 November 2016 